\title{
Successful Endoscopic Removal of a Transmural Internally Migrating Gossypiboma
}

\author{
Ashish Agarwal ${ }^{10}$ Ashish Chauhan ${ }^{10}$ Sanchit Sharma ${ }^{10}$ Kumble Seetharama Madhusudhan ${ }^{2(1)}$ \\ Rajesh Panwar ${ }^{3}$ (1)
}

${ }^{1}$ Department of Gastroenterology and Human Nutrition, All India Institute of Medical Sciences, New Delhi, India

2 Department of Radiology, All India Institute of Medical Sciences, New Delhi, India

${ }^{3}$ Department of Gastrointestinal Surgery and Liver Transplantation, All India Institute of Medical Sciences, New Delhi, India

Address for correspondence Rajesh Panwar, MS, MCh, Department of Gastrointestinal Surgery and Liver Transplantation, All India Institute of Medical Sciences, Ansari Nagar, New Delhi 110029, India (e-mail: rajeshpanwar81@gmail.com).

J Digest Endosc 2022;13:58-60.

\begin{abstract}
Keywords

- gossypiboma

- endoscopic removal

- complications

An accidentally retained sponge inside the body of a patient (gossypiboma) is a rare and serious adverse event after a surgical procedure with serious medicolegal implications and complications. It is commonly associated with abdominal surgeries with cholecystectomy most commonly implicated. Whorl-like appearance or mottled translucencies and radiopaque marker on imaging is diagnostic. Transmural migration into a hollow viscous has been infrequently reported. The preferred approach for the removal of gossypiboma is surgery. However, in cases of complete transmural migration, endoscopic removal remains a viable option.
\end{abstract}

\section{Introduction}

Gossypiboma (or textiloma or gauzeoma) represents a gauze or sponge that has been left unintentionally inside the body of a patient after a surgical procedure. Although uncommon, it is a serious adverse event associated with significant morbidity and mortality. We hereby present a case of successful endoscopic removal of gossypiboma.

\section{Case Report}

A 35-year-old female presented to our hospital with complaints of pain in the right hypochondrium with a feeling of lump for the past 1 month with recurrent nonbilious vomiting immediately after meals for 20 days. There was no history of fever or jaundice. The patient had undergone attempted cholecystectomy for symptomatic gallbladder stones in an- other hospital one and half months back. The surgical notes revealed that the surgeon had noted significant adhesions around the gallbladder and abandoned the procedure.

Investigations showed normal liver function tests and contracted gallbladder on abdominal ultrasonography. Common bile duct was normal. The initial contrast-enhanced computed tomography (CT) scan of the abdomen done 20 days prior to the presentation to our hospital showed a thick-walled lesion anterior to the pyloric antrum of the stomach with mottled air lucencies, suggestive of gossypiboma (-Fig. 1). The stomach wall was thickened. There were hyperdense areas within the sponge, suggestive of hemorrhage. Esophagogastroduodenoscopy (EGD) was done at our hospital that showed a gauze inside the body and the antrum of the stomach, extending up to the duodenal bulb causing obstruction (-Fig. 2). Endoscope was negotiable across the duodenal bulb into the second part of the duodenum.
DOI https://doi.org/ 10.1055/s-0040-1717826. ISSN 0976-5042. (c) 2022. Society of Gastrointestinal Endoscopy of India. All rights reserved.

This is an open access article published by Thieme under the terms of the Creative Commons Attribution-NonDerivative-NonCommercial-License, permitting copying and reproduction so long as the original work is given appropriate credit. Contents may not be used for commercial purposes, or adapted, remixed, transformed or built upon. (https://creativecommons.org/ licenses/by-nc-nd/4.0/)

Thieme Medical and Scientific Publishers Pvt. Ltd., A-12, 2nd Floor, Sector 2, Noida-201301 UP, India 



Fig. 1 Axial (A) and coronal reformat, (B) computed tomography images (done 20 days before presentation) showing a thick-walled lesion with mottled air foci (arrows), located anterior to thickened pyloric antrum of the stomach (arrowhead). Gallbladder shows calculi and edematous wall (asterisk).

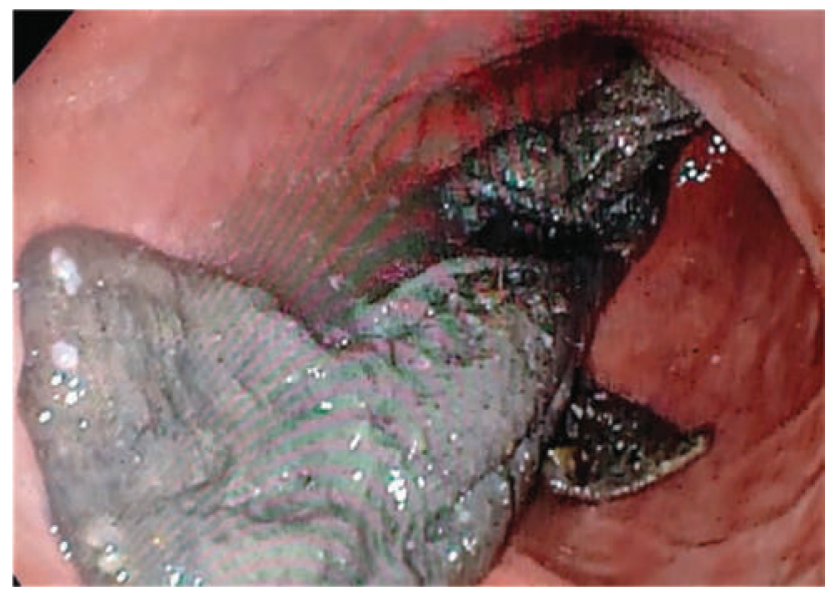

However, intraperitoneal extension of the gauze could not be ascertained at endoscopy and thus retrieval was not attempted. A repeat CT scan done at our institution showed near complete migration of the sponge into the stomach through a fistulous opening in the distal gastric antrum (-Fig. 3). EGD was repeated and the gauze was noted to be adherent to the duodenal bulb from where it was first disimpacted by pulling at the distal end with a rat-tooth forceps. Once the gauze was disimpacted, it was held at the proximal end with the rat-tooth forceps and was pulled out. There was mild difficulty in negotiating the gauze at the upper esophageal sphincter but could be managed with a constant firm pull. After the removal of the gauze ( - Fig. 4), a small superficial ulcer with mild mucosal bleed was observed in the duodenal bulb that was the likely site of the internal migration of the gauze (-Fig. 5). A nasogastric tube was placed for continuous aspiration and the patient was kept fasting for 48 hours. A broad-spectrum antibiotic was given for 3 days. On day 3, EGD showed almost completely healed duodenal mucosa. Patient was discharged home with a plan for cholecystectomy after 3 months.

\section{Discussion}

Although the exact incidence of intrabdominal gossypiboma is not known due to frequent under reporting of the condi-

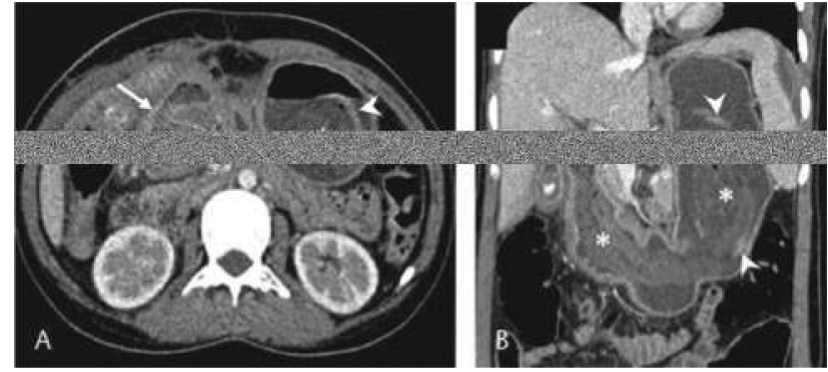

Fig. 3 Axial (A) and coronal (B) computed tomography (CT) images ( $C T$ done prior to endoscopic retrieval) showing complete migration of the surgical sponge into the stomach (asterisks) with hyperdense areas (arrowheads). A fistulous opening is seen in the distal antrum (arrow in $\mathrm{A}$ ) indicating the site of entry.

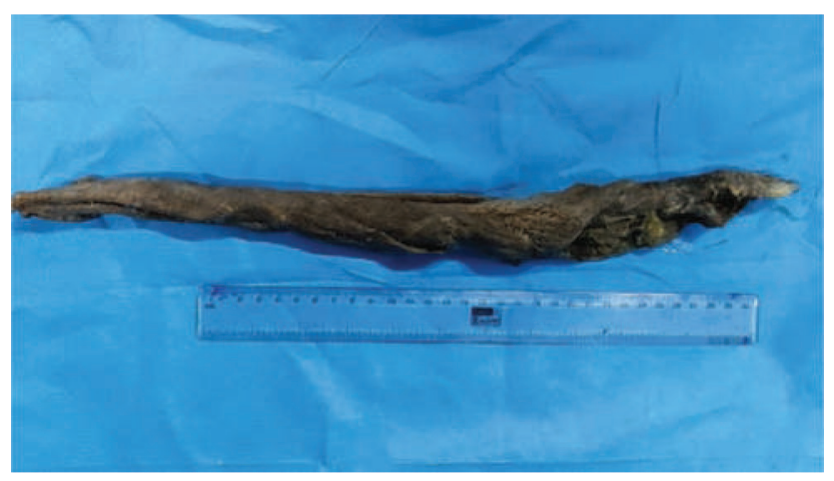

Fig. 4 The gossypiboma after removal.

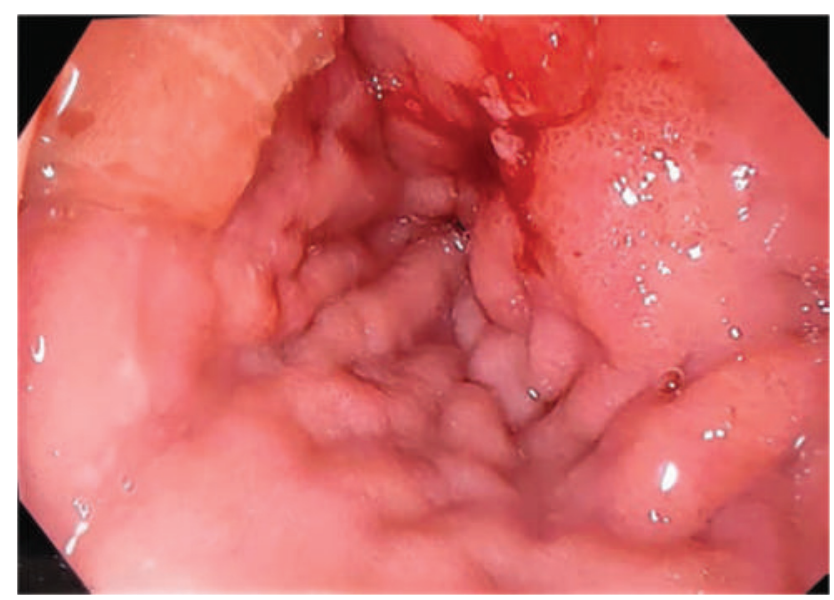

Fig. 5 Endoscopic image showing a small superficial ulcer with mild ooze from the duodenal bulb after removal of the gossypiboma.

tion because of associated medicolegal implications, studies have reported an incidence of 1 in every 1,000 to 1,500 abdominal surgeries. ${ }^{1}$ Cholecystectomy is the most common surgery implicated for gossypiboma followed by gynecologic surgeries. Previous studies have shown increased incidence of retention of foreign body after surgery in emergencies, unplanned changes in procedure, and in patients with higher body mass index. ${ }^{2}$ 
The retained gossypiboma can incite a fibrotic reaction leading to adhesions, encapsulation, and eventually calcification, disruption, or partial absorption or diffusion. In other cases, it can incite an exudative inflammatory type of reaction with abscess formation. The abscess can cause pressure and erode the surrounding organs, and can lead to a foreign body sinus, enterocutaneous fistula formation, or transmigration into the gut lumen. ${ }^{3}$ Migration of the retained sponge is less common compared with abscess formation. Inflammation followed by the necrosis of bowel wall as a result of inflammation results in transmural migration of the retained gauze into the bowel. Most common site of transmigration is the small intestine due to its thin wall and larger surface. Only a few cases of migration into the duodenal lumen have been described so far. ${ }^{4}$

The diagnosis is relatively easy with the help of a CTscan in a symptomatic patient with a history of surgery. Radiopaque marker and mottled radiolucencies or whorl-like appearance on a plain X-ray may also point toward a gossypiboma. Crosssectional imaging (CT and magnetic resonance imaging) shows presence of multiple spongiform air pockets inside a soft tissue lesion or whorl-like appearance and a radiopaque marker, if present. However, it is frequently misdiagnosed as a neoplasm, abscess, or intrabdominal hematoma, thus leading to radical surgical interventions. Hence, a gossypiboma should be kept as a differential in any postoperative patient presenting with suspicious imaging findings.

Gossypiboma should be removed soon after the diagnosis. Open or laparoscopic surgical removal is the preferred approach depending on the size and location of the gossypiboma and the skills of the surgeon. ${ }^{5}$ In cases with intraluminal migration of the gossypiboma, with no evidence of free perforation or bleeding, complete endoscopic approach can also be attempted for the removal of gossypiboma as was successfully done in the present case. ${ }^{4}$

To conclude, gossypiboma is a rare and serious adverse event and mostly requires surgery. Endoscopic removal can be attempted in cases with transmural migration inside the gastrointestinal tract.

\section{Funding}

None.

Conflict of Interest

None declared.

\section{Acknowledgment}

We would like to acknowledge our endoscopy nursing staff for all their support.

\section{References}

1 Hyslop JW, Maull KI. Natural history of the retained surgical sponge. South Med J 1982;75(06):657-660

2 Gawande AA, Studdert DM, Orav EJ, Brennan TA, Zinner MJ. Risk factors for retained instruments and sponges after surgery. $\mathrm{N}$ Engl J Med 2003;348(03):229-235

3 Wattanasirichaigoon S. Transmural migration of a retained surgical sponge into the intestinal lumen: an experimental study. J Med Assoc Thai 1996;79(07):415-422

4 Lv Y-X, Yu C-C, Tung C-F, Wu C-C. Intractable duodenal ulcer caused by transmural migration of gossypiboma into the duodenum-a case report and literature review. BMC Surg 2014;14:36

5 Obeidat KA, Aleshawi AJ, Alebbini MM, Bani Yasin SN. Abdominal intraluminal gossypiboma: demographics, predictors of intraluminal site, diagnostic and treatment measures. Clin Exp Gastroenterol 2020;13:65-72 